\title{
Cell Decompositions of the Projective Plane with Petrie Polygons of Constant Length
}

\author{
J. Bokowski, ${ }^{1}$ J.-P. Roudneff, ${ }^{2}$ and T.-K. Strempel $^{1}$ \\ ${ }^{1}$ Department of Mathematics, University Darmstadt, \\ Schloßgartenstrasse 7, D-64289 Darmstadt, Germany \\ bokowski@mathematik.th-darmstadt.de \\ ${ }^{2} 85-87$ ave du General Lederc, \\ F-78220 Viroflay, France
}

\begin{abstract}
We study dual pairs of combinatorial face-to-face cell decompositions $\left(\mathcal{D}_{P^{2}}, \mathcal{D}_{P^{2}}^{*}\right)$ of the real projective plane $P^{2}$ such that their canonically induced cell decompositions $\left(\mathcal{D}_{S^{2}}, \mathcal{D}_{S^{2}}^{*}\right)$ on the 2-sphere $S^{2}$ form dual pairs of combinatorical types of convex polyhedra, and such that these dual pairs share two natural properties with those induced by dual pairs of Platonic solids: (1) Every Petrie polygon is a finite simple closed polygon of length $2(n-1)$ for some fixed $n$. (2) Every pair of Petrie polygons has precisely two common edges. Such pairs of face-to-face cell decompositions of the projective plane are in one-to-one correspondence with $n$-element pseudoline arrangements. We study in particular those dual pairs of cell decompositions $\left(\mathcal{D}_{P^{2}}, \mathcal{D}_{P^{2}}^{*}\right)$ in which $\mathcal{D}_{P^{2}}$ or $\mathcal{D}_{P^{2}}^{*}$ has only 3 -valent vertices, i.e., via the above one-to-one correspondence: $p_{3}$-maximal pseudoline arrangements. A $p_{3}$-maximal pseudoline arrangement with $n$ elements in turn determines a neighborly 2-manifold with Euler characteristic $\chi=n(7-n) / 6$, and vice versa, this neighborly 2-manifold uniquely determines its generating $p_{3}$-maximal pseudoline arrangement. We provide new inductive constructions for finding infinite example classes of $p_{3}$-maximal pseudoline arrangements from small existing ones, we describe an algorithm for generating them, we provide a complete list of existence up to $n=40$, and we discuss their properties.
\end{abstract}

\section{Introduction}

Connections between different areas in mathematics are very often fruitful. Seeing a given mathematical structure from a different point of view, together with the transition of ideas into a new context, induces creative developments. Within the theory of oriented matroids, we already know many surprising connections. In the study of line arrangements, we have a connection to algebraic surfaces, compare [13] with [3]. 


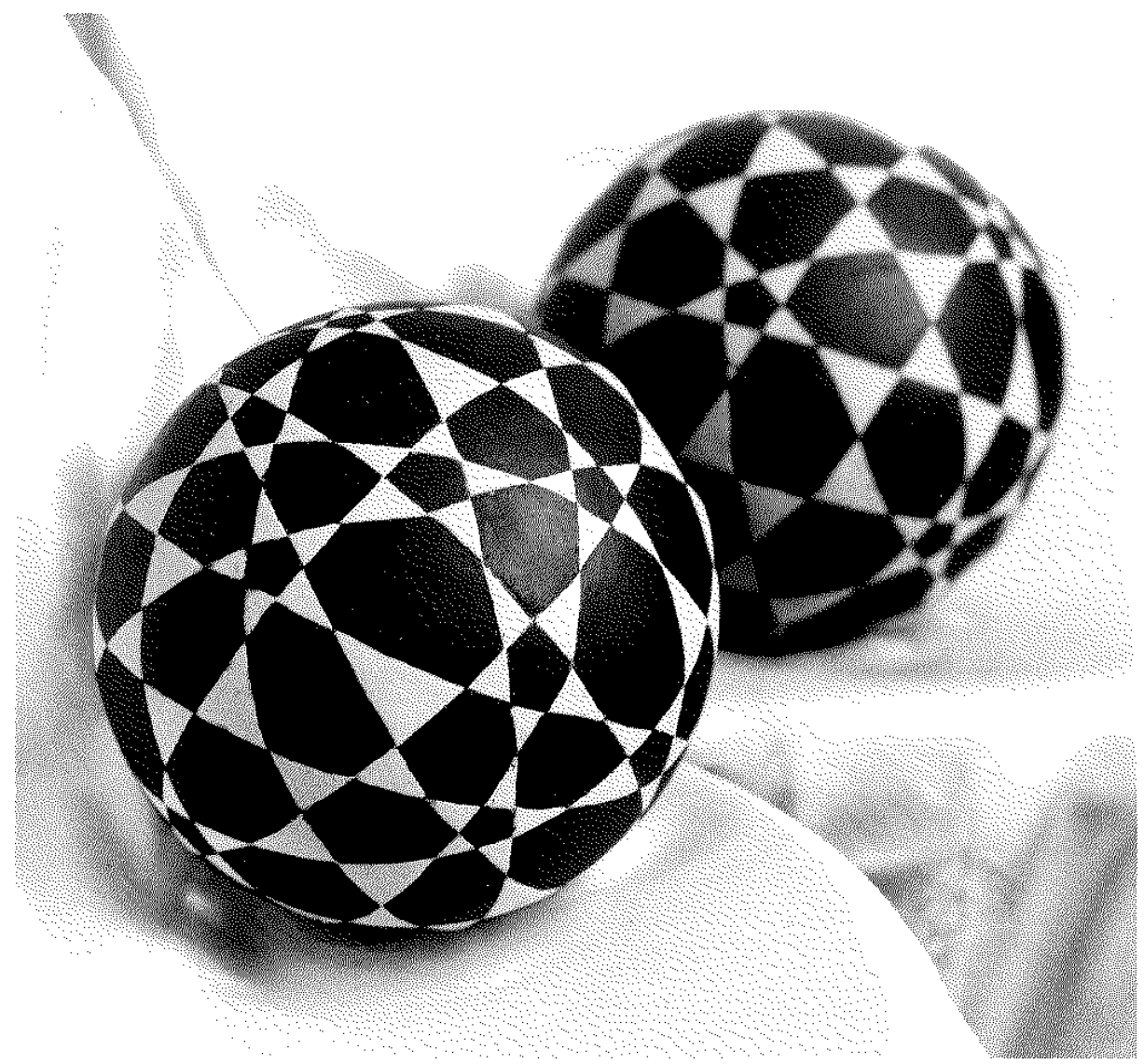

Fig. 1. The depicted 1-sphere system on the 2-sphere represents not only a $p_{3}$-maximal pseudoline arrangement when antipodal points are identified, but also a Petrie-decomposition via Theorem 2.2, a neighborly 2-manifold via Theorem 2.3, and a special block design: a so-called Mendelsohn triple system.

Within this article we describe such bridges between the study of line and pseudoline arrangements on the one hand and the concepts of Petrie polygons, neighborly manifolds, and block designs on the other. We shall see, for example, that the picture in Fig. 1 (pottery model of the first author) also represents a neighborly 2-manifold.

One of the combinatorial generalizations of the study of Platonic solids leads to the theory of regular maps on orientable 2-manifolds with Petrie polygons of constant length. For a survey on modern developments in regular polytopes see the article by McMullen [17] and related articles in the same volume. Compare also the survey article by Brehm and Wills in [7] and the article by McMullen and Schulte within this volume [18].

We study face-to-face cell decompositions of the real projective plane $P^{2}$ as the first example of a nonorientable 2-manifold. Such a cell decomposition $\mathcal{D}_{P^{2}}$ of the real projective plane leads in a canonical way to an induced cell decomposition $\mathcal{D}_{S^{2}}$ of the 2-sphere $S^{2}$, and it can be compared with the cell decomposition of the 2-sphere induced by a Platonic solid. 
Definition 1.1. We define a Petrie polygon, compare [8, p. 24], of an abstract finite face-to-face cell decomposition of a combinatorial 2-manifold to be a (possibly infinite) abstract polygon such that every two consecutive abstract edges, but not three, belong to one cell, see Fig. 7 where we have depicted a Schlegel diagram for each Platonic solid with all its Petrie polygons.

Given a face-to-face decomposition of a combinatorial 2-manifold, then every two adjacent edges of a face determine a unique Petrie polygon, which in general will be infinite. Conversely, every Petrie polygon arises in this way.

Definition 1.2. We call a dual pair of abstract face-to-face cell decompositions $\left(\mathcal{D}_{P^{2}}, \mathcal{D}_{P^{2}}^{*}\right)$ of the real projective plane a Petrie decomposition when its induced pair of cell decompositions $\left(\mathcal{D}_{S^{2}}, \mathcal{D}_{S^{2}}^{*}\right)$ on the 2-sphere forms a dual pair of combinatorical types of convex polyhedra and when it shares the following two properties with dual pairs of Platonic solids:

(1) Every Petrie polygon is a finite simple closed polygon of length $2(n-1)$ for some fixed $n$.

(2) Every pair of Petrie polygons of $\mathcal{D}_{S^{2}}$ or of $\mathcal{D}_{S^{2}}^{*}$ has precisely two common edges.

We show in Section 2, Theorem 2.1, that Petrie decompositions $\left(\mathcal{D}_{P^{2}}, \mathcal{D}_{P^{2}}^{*}\right)$ with Petrie polygon length $2(n-1)$ are in one-to-one correspondence with reorientation classes of uniform oriented matroids in rank 3 with $n$ elements. These reorientation classes are equivalent to (unoriented) pseudoline arrangements in the real projective plane which were introduced by Levi in [16] (see also the research monograph by Grünbaum [13]). Pseudoline arrangements have received tremendous interest during the last 20 years when connections to many other areas in mathematics were discovered (see [4] and [5]). We study in particular those Petrie decompositions in which one part, say $\mathcal{D}_{P^{2}}$, has only 3 -valent vertices, in other words, via the above one-to-one correspondence: $p_{3}$-maximal pseudoline arrangements, see Theorem 2.2. These special pseudoline arrangements have been studied by Grünbaum, Roudneff, Harborth, and others. A $p_{3}$-maximal pseudoline arrangement with $n$ elements in turn determines a neighborly 2-manifold with Euler characteristic $\chi=n(7-n) / 6$, and vice versa, this neighborly 2-manifold uniquely determines its generating $p_{3}$-maximal pseudoline arrangement, see Theorem 2.3. This correspondence was also observed by Coxeter in the context of regular maps.

In Section 3 we provide new inductive constructions for finding classes of infinite classes of examples from small existing ones. In Section 4 we describe an algorithm for generating $p_{3}$-maximal pseudoline arrangements which leads in particular to uniqueness assertions for small numbers of elements $n$. Finally, in Section 5, we provide a complete list of those $n, n \leq 40$, for which there exists at least one $p_{3}$-maximal pseudoline arrangement with $n$ elements. For basic notions of oriented matroids, we refer the reader to [4].

\section{Petrie Decompositions and Pseudoline Arrangements}

In this section we formulate equivalence assertions similar to the example in Fig. 8. The left cell decomposition defines a Petrie decomposition which corresponds to an 
Archimedean body and which gives rise, on the one hand, to a corresponding $p_{3}$-maximal pseudoline arrangement (depicted in the middle) and, on the other hand, to six Möbius strips defining the corresponding neighborly 2-manifold with Euler characteristic $\chi=$ -5 . The five triangles of each Möbius strip consist of a vertex of its 5-gon together with its two adjacent vertices.

Definition 2.1. A pseudoline arrangement in the projective plane $P^{2}$ with $n$ elements is an ordered set $\left\{p_{1}, \ldots, p_{n}\right\}, n \geq 3$, of simple, piecewise linear, closed curves in $P^{2}$, so that any two curves have exactly one point in common in which they cross. An arrangement is called simple if every intersection point belongs to exactly two pseudolines.

Definition 2.2. A pseudoline arrangement with $n$ elements is called $p_{3}$-maximal if its number of triangles is $n(n-1) / 3$, which is the theoretical upper bound for the number of triangles. Thus, if it is attained, it is the maximum number of triangles.

A simple pseudoline arrangement in the projective plane can be considered on the 2-sphere as a 1-sphere system. It defines a cell decomposition on the 2-sphere which is 2-colorable. For a given simple pseudoline arrangement (the nonsimple case leads to a degeneration), we define a pair of dual graphs on the 2-sphere (one for each color) by putting a point in each cell and by connecting two points if and only if the corresponding cells have equal colors and a common vertex. This pair of dual graphs defines a pair of dual cell decompositions for which we consider its Petrie polygons.

Theorem 2.1. Petrie decompositions $\left(\mathcal{D}_{P^{2}}, \mathcal{D}_{P^{2}}^{*}\right)$ with the Petrie polygon length $n-1$ are in one-to-one correspondence with simple pseudoline arrangements with $n$ elements, $n \geq 4$.

Proof of Theorem 2.1. The fact that simple pseudoline arrangements define a Petrie decomposition follows from the above considerations (e.g., the cell decomposition is 2-colorable) together with basic properties of pseudoline arrangements. We now consider the two properties in Definition 1.2. (1) is equivalent to (1a) and (1b) in what follows. For (1a) (all Petrie polygons of $\mathcal{D}_{S^{2}}$ and $\mathcal{D}_{S^{2}}^{*}$ have the equal length $2(n-1)$ ), we consider the set of cocircuits on a given pseudoline; property (2) (every pair of Petrie polygons of $\mathcal{D}_{S^{2}}$ or of $\mathcal{D}_{S^{2}}^{*}$ has precisely two common edges) reflects the fact that any two pseudolines cross precisely once; and property (1b) (no Petrie polygon of $\mathcal{D}_{S^{2}}$ or of $\mathcal{D}_{S^{2}}^{*}$ has a self-intersection) holds because pseudolines have no self-intersections. We obtain combinatorial types of convex polyhedra because the cell decomposition on the 2-sphere $(n \geq 4)$ has as its 1 -skeleton a planar 3-connected graph and because of the theorem of Steinitz (see, e.g., [24]).

In the opposite direction we consider a given Petrie decomposition. We start with a dual pair of face-to-face cell decompositions $\left(\mathcal{D}_{P^{2}}, \mathcal{D}_{P^{2}}^{*}\right)$ with properties (1) and (2) of Definition 1.2. We apply the inverse construction of the foregoing definition of the pair of dual graphs to obtain a pseudoline for each Petrie polygon. The required properties for these pseudolines (see Definition 2.1) follow from Definition 1.2. 
Theorem 2.2. Petrie decompositions for which one part has only 3-valent vertices are in one-to-one correspondence with $p_{3}$-maximal pseudoline arrangements.

Proof of Theorem 2.2. Once the transition from simple pseudoline arrangements to Petrie decompositions and vice versa has been established, we see that the property of 3-valent vertices of one of the components of the Petrie decomposition is equivalent to the fact that we have alternatingly combinatorial triangles (or mutations) along each pseudoline; in other words, our simple pseudoline arrangement is a $p_{3}$-maximal one.

Theorem 2.3. From each $p_{3}$-maximal pseudoline arrangement a unique neighborly 2-manifold can be obtained in a straightforward manner, and, moreover, neighborly 2-manifolds obtained in this way contain all the data for the $p_{3}$-maximal pseudoline arrangement. The neighborly 2-manifolds obtained are nonorientable ones.

Proof of Theorem 2.3. For a given $p_{3}$-maximal pseudoline arrangement, we define each pseudoline to form a point and we define a triple of three pseudolines $(i, j, k)$ to form a triangle whenever the pseudoline arrangement has a triangle (a mutation) which is bounded by the pseudolines $i, j$, and $k$. We next claim that our set of triangles forms a neighborly nonorientable 2-manifold of Euler characteristic $\chi=n(7-n) / 6$.

All triangles incident with a fixed point $p$ are those adjacent to a fixed pseudoline. However, these triangles are arranged in a circular order which shows that the star around $p$ is a 2-disk. We have to show that each edge lies in precisely two triangles: the edges of triangles correspond to cocircuits (points of intersections of pseudolines) and we observe that all possible edges belong to our set of edges defined by the triangles and, moreover, for each "edge" (= point of intersection), there are precisely two adjacent triangles within the pseudoline arrangement which yields the 2-manifold property.

Obtaining a manifold when considering (in view of Theorem 2.2) the "Petrie polygons" (= pseudolines) as points, was found by the third author independently of what Coxeter [9] observed in the context of regular maps, see also a recent survey by McMullen [17, p. 99].

By using standard combinatorial arguments, it can be shown that every $p_{3}$-maximal pseudoline arrangement contains at least a 5-gon (actually even at least three), compare [20]. We look at such a 5-gon and its five adjacent triangles and we observe that they form a Möbius strip. Our 2-manifold must be a nonorientable one, its Euler characteristic can be evaluated from the combinatorics of $p_{3}$-maximal pseudoline arrangements: $\chi=$ $n(7-n) / 6$.

Block Designs. The connection between neighborly pseudomanifolds and Mendelsohn triple systems has been studied in [2]. Our neighborly 2-manifolds are in particular pseudomanifolds, which leads to the same connection similar to that described in this article. Every $p_{3}$-maximal pseudoline arrangement with $n$ elements defines a $2-(n, 3,2)$ block design, a Mendelsohn triple system. 


\section{Inductive Constructions for Generating $p_{3}$-Maximal Pseudoline Arrangements}

The problem of finding examples of $p_{3}$-maximal arrangements of pseudolines has actually received only partial solutions. The general upper bound $p_{3} \leq n(n-1) / 3$ is true for $n \geq 9$, see [21]. Moreover, for simple arrangements, equality holds if and only if the triangles alternate along each pseudoline, which requires $n$ to be even. The condition $n \neq 12$ and the evident congruence $n \neq 2 \bmod 3$ are also necessary, see [14] and [19]. Infinite classes of such arrangements are known but the problem of sufficiency of the preceding conditions is still unsolved, the first open case being $n=48$. Starting from a $p_{3}$-maximal arrangement of $n$ pseudolines, several methods are known to deduce $p_{3}$-maximal arrangements of $2 n-2$, or $3 n-2$ pseudolines, respectively, see [14] and [19].

The question by Harborth in [14] about the stretchability of a particular arrangement led to a first example and an infinite sequence of nonstretchable $p_{3}$-maximal arrangements, see [6]. The converse has recently been shown as well; one of the mentioned constructions can be specialized in order to preserve realizability, in other words: there exists an infinite family of stretchable $p_{3}$-maximal arrangements (see [11]).

In this section we present two new recursive constructions which yield $p_{3}$-maximal arrangements with $7 n-6$ and $8 n-8$ pseudolines from a $p_{3}$-maximal arrangement $\mathcal{A}$ of $n$ pseudolines. The special role of one element in the second construction leads in general to as many nonisomorphic new $p_{3}$-maximal arrangements as there are different elements (considering the automorphism group of the underlying arrangement and its fundamental region) in the given $p_{3}$-maximal arrangement.

We first present the transformation $n \rightarrow 7 n-6$ for $n \geq 6$. The method consists of selecting a pseudoline $L_{\infty}$ in $\mathcal{A}$ (considered as the line at infinity allowing an orientation of the affine plane $P^{2} \backslash L_{\infty}$ ) and adding to every pseudoline $L$ of $\mathcal{A} \backslash L_{\infty}$ six pseudolines which are near parallel to $L$, i.e., which cross each other near $L_{\infty}$. More precisely, Fig. 9 shows in a graphical way how a face $F$ of $\mathcal{A}$ is dissected, according to the fact that $F$ is bounded by $L_{\infty}$ or not. On the figures the construction (the new curves are colored) is given for faces with three, five, and six edges, the generalization to $p \geq 7$ being immediate. We notice that a $p_{3}$-maximal arrangement $\mathcal{A}$ has no quadrilaterals, except in the case $n=4$ for which a variant of the construction is possible leading to a $p_{3}$-maximal arrangement $\mathcal{A}^{\prime}$ with 22 elements, compare Fig. 2.

The fact that the global set $\mathcal{A}^{\prime}$ of dissected faces forms a $p_{3}$-maximal pseudoline arrangement now follows from geometric considerations, any two curves of $\mathcal{A}^{\prime}$ crossing exactly once and no two curves of $\mathcal{A}^{\prime}$ having a self-intersection. It can also be verified (in a combinatorial way) that the number of vertices of $\mathcal{A}^{\prime}$ is exactly $(7 n-6)(7 n-7) / 2$, so that it suffices to remark that two curves of $\mathcal{A}^{\prime}$ cross at least once. Since the triangles of $\mathcal{A}^{\prime}$ alternate by construction, we have established that $\mathcal{A}^{\prime}$ is a $p_{3}$-maximal arrangement with $7 n-6$ elements.

For the transformation $n \rightarrow 8 n-8$ the method is similar: we replace each pseudoline $L$ of $\mathcal{A} \backslash L_{\infty}$ by eight pseudolines which are near parallel to $L$. After the construction, the pseudolines of $\mathcal{A}$ are removed. Figure 10 describes again the process in a graphical way. 


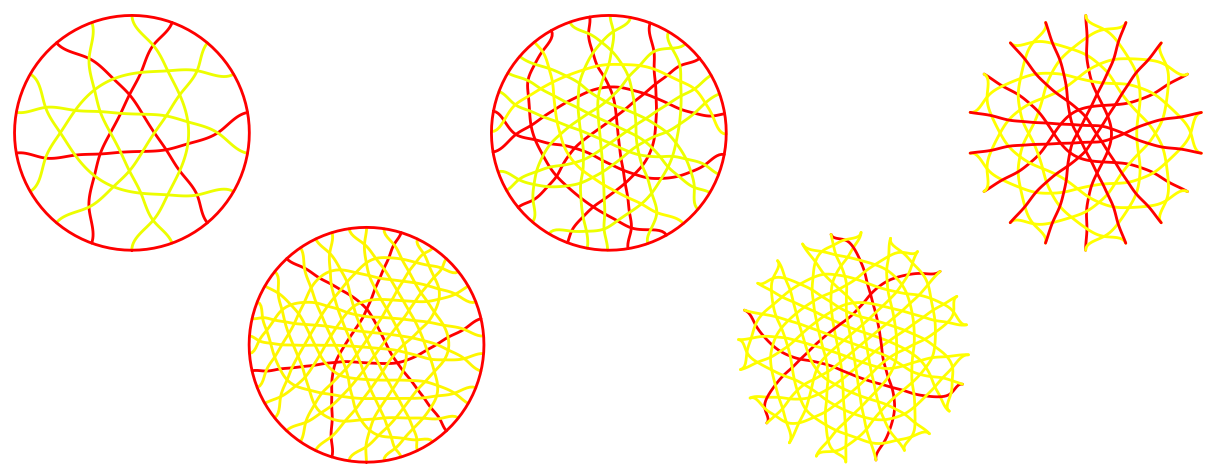

Fig. 2. Examples of known constructions.

\section{An Algorithm for Generating $p_{3}$-Maximal Pseudoline Arrangements}

We describe an algorithm for generating all $p_{3}$-maximal pseudoline arrangements with $n=2 m$ elements and $n(n-1) / 3$ triangles.

For a $p_{3}$-maximal pseudoline arrangement with $n$ elements, we consider its FolkmanLawrence representation and its representation as a hyperline sequence [5]. We obtain geometric ideas from looking at the Folkman-Lawrence representation, whereas for the formal algorithmical description we pursue the steps by looking at a normalized hyperline sequence, i.e., the list of all oriented rank 2 contractions.

A $p_{3}$-maximal pseudoline arrangement has a sequence of $(n-1)$ alternating triangles along each pseudoline. We use this property for its algorithmical construction. In particular, this property can be assumed for line $n$ (which we assume to be the line at infinity when we draw the Folkman-Lawrence representation).

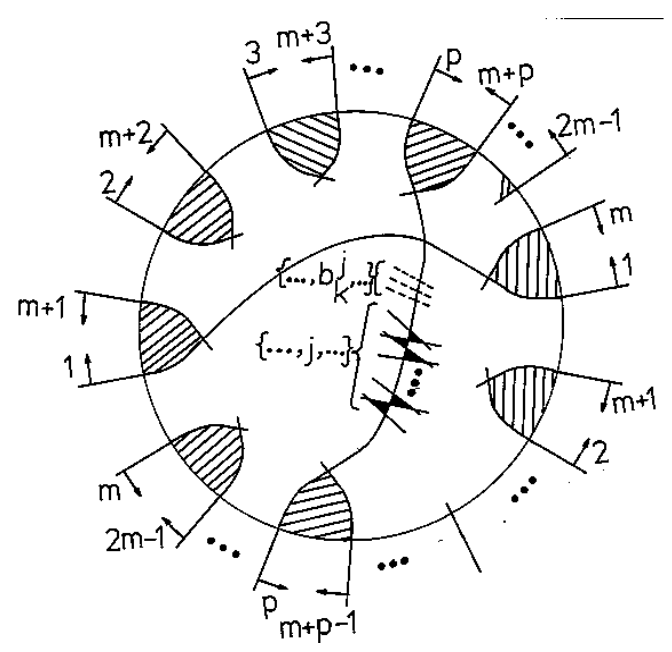

Fig. 3. Labeling for the algorithmic generation. 
We start with such a circular sequence of alternating triangles at line $n$ for generating all possible $p_{3}$-maximal pseudoline arrangements for given $n=2 m$, see Fig. 3 . We choose orientations for our pseudolines $1, \ldots, n-1$ such that we get a circular structure along pseudoline number $n$, compare Fig. 3 and the first line in the following table. This circular structure can be expressed with the standard modulo function: in a general hyperline $p, p \in\{1, \ldots, 2 m-1\}$, we consider all numbers with absolute value modulo $2 m-1$. In addition we use the sign to indicate the orientation (see the table). Our initial data structure written as a corresponding set of partial hyperline sequences together with boolean variables $\triangle_{p}^{l},\left\{c l_{p}^{j}, \triangle_{p, j}^{m l}, \triangle_{p, j}^{m r}, c r_{p}^{j}\right\}, \triangle_{p}^{r}$ for $p \in\{1, \ldots, n-1\}$ reads as follows:

\begin{tabular}{|c|c|c|c|c|c|c|}
\hline$n$ & +1 , & $-m-1$ & +2 & $-m-2, \ldots$ & $-2 m+1$ & $m$ \\
\hline 1 & $(-n$, & $-m-1$ & $\Delta_{1}^{l}$ & $\{(., .,\{\},.\{\},.\{\},. .,)\}$. & $\left(\triangle_{1}^{r}\right.$, & $m)$ \\
\hline 2 & $(-n$, & $-m-2$ & $\Delta_{2}^{t}$ & ),$\{(., .,\{\},.\{\},.\{\},. .,)\}$. & $\left(\triangle_{2}^{r}\right.$ & $m+1)$ \\
\hline : & & : & & & & $\cdot$ \\
\hline$p$ & $(-n$, & $-m-p$ & $\triangle_{p}^{l}$ & ),$\quad\left\{\left(c l_{p}^{j}, \triangle_{p, j}^{m l},\left\{b_{k}^{j}\right\}\{j\}\left\{a_{l}^{j}\right\}, \triangle_{p, j}^{m r}, c r_{p}^{j}\right)\right\}$ & $\left(\triangle_{p}^{r}\right.$ & $m-1+p)$ \\
\hline : & $(-n$, & $\begin{array}{l}: \\
-1,\end{array}$ & $\left.\triangle_{m}^{l} \quad\right)$, & ),$\quad\{(., .,\{\},.\{\},.\{\},. . .)\}$, & $\left(\triangle_{m}^{r}\right.$ & $\begin{array}{c}: \\
2 m-1)\end{array}$ \\
\hline$m+1$ & $(-n$, & -2 & $\left.\Delta_{m+1}^{l}\right)$, & ),$\{(., .,\{\},.\{\},.\{\},. . .)\}$. & $\left(\triangle_{m+1}^{r}\right.$, & 1) \\
\hline$m+2$ & $(-n$, & -3 & $\left.\Delta_{m+2}^{l+1}\right)$, & ),$\{(., .,\{\},.\{\},.\{\},. .,)\}$. & $\left(\triangle_{m+2}^{r+1}\right.$ & 2) \\
\hline $2 m-1$ & $(-n$, & $-m$, & $\left.\Delta_{2 m-1}^{l}\right)$, & ),$\quad\{(., .,\{\},.\{\},.\{\},. . .)\}$. & $\left(\triangle_{2 m-1}^{r}\right.$, & $m-1)$ \\
\hline
\end{tabular}

We have introduced the boolean variable $\triangle_{p}^{l}$ to indicate whether or not the last triangle on the left side is in an upright position and $\triangle_{p}^{r}$ indicates the corresponding position of the triangle on the right within line $p$. For all the remaining elements $j$ (so far not involved in the construction of the first set of triangles adjacent to line $n$ ) crossing pseudoline number $p$, we assume that we have marked in the set left of $\{j\}$ all elements $b_{k}^{j}$ which have to cross line $p$ left of $j$; similarly, $a_{l}^{j}$ is an element which has to cross line $p$ right of $j$. We obtain this information from the oriented matroid property since several signed bases are known from the very beginning.

In order to describe the remaining data structure, we consider a general inductive step of our algorithm to generate $p_{3}$-maximal pseudoline arrangements in which we already know a certain set of triangles together with a set of signed bases of the partial oriented matroid as a representative for our $p_{3}$-maximal pseudoline arrangement. In this general case, because of the triangle structure, in general the former set $\{j\}$ has been replaced with an ordered set $\{\ldots, j, \ldots\}$ of elements corresponding to a sequence of adjacent triangles within line $p$, or the set $\{j\}$ has in the meantime been glued perhaps together with other elements to one of the sides. The known triangle types left and right for the set $\{\ldots, j, \ldots\}$ (when its cardinality exceeds 1 ) are stored as boolean variables $\triangle_{p, j}^{m l}$ and $\triangle_{p, j}^{m r}$.

The signed bases known so far determine for each set $\{\ldots, j, \ldots\}$ of elements a set of left neighbors (before $j)\left\{\ldots, b_{k}^{j}, \ldots\right\}$ (and right neighbors (after $j$ ) $\left\{\ldots, a_{k}^{j}, \ldots\right\}$, 
respectively). For each set $\{\ldots, j, \ldots\}$ with its pair of sets $\left(\left\{\ldots, b_{k}^{j}, \ldots\right\},\left\{\ldots, a_{k}^{j}, \ldots\right\}\right)$ on the left and on the right of $\{\ldots, j, \ldots\}$, we introduce the boolean variable $c l_{p}^{j}$ which is true when we can glue the set $\{\ldots, j, \ldots\}$ to the left, i.e., the left set $\left\{\ldots, b_{k}^{j}, \ldots\right\}$ is empty and in case the cardinality of $\{\ldots, j, \ldots\}$ is greater than 1 , the triangle types $\triangle_{p}^{l}$ and $\triangle_{j}^{m l}$ are equal. Thus $c l_{p}^{j}$ tells us whether or not our set (containing $j$ ) is a candidate for gluing it to the left. In a similar way the boolean variable $c r_{p}^{j}$ tells us whether or not our set is a candidate for gluing it to the right.

Starting with this data structure, we count the number of candidates for gluing them either to the left or to the right. We determine the smallest index of a pseudoline with a minimal number of candidates (preferable left candidates) and we glue all these candidates one after the other to their boundary. Each case defines a new triangle, which reduces the freedom within the remaining data structure. The new triangle can be inserted in two additional lines, new signed bases are known in general when comparing the glued sequence of elements of $\{\ldots, j, \ldots\}$ with all remaining elements in this line. In general these signs change the sets $\left\{\ldots, b_{k}^{j}, \ldots\right\}$ and $\left\{\ldots, a_{k}^{j}, \ldots\right\}$. When all consequences have been used to change the data structure accordingly, we repeat our algorithm as long as we find candidates. When we get a completed scheme without contradictions, our data structure defines a $p_{3}$-maximal pseudoline arrangement.

During our algorithmic generation, we use the following observations. The sign of an element $q$ within the general line $p$ is known because, for $p=n$ and all $q, \operatorname{sign}(q)$ is defined as seen in the first line in the scheme, for all other $p$ and $q=n$, the sign is negative, for $p, q \in\{1, \ldots, 2 m-1\}$, we have from line $p$ and because $\chi$ is an alternating function $\chi(p, n, q)=-\operatorname{sign}(q)=-\chi(n, p, q)$. For $p, q \in\{1, \ldots, m\}$ or $(p-m),(q-m) \in\{1, \ldots, m-1\}$, we have $\chi(n, p, q)=+1$ for $p \leq q$ and otherwise $\chi(n, p, q)=-1$. For $p \in\{1, \ldots, m\}$ and $(q-m) \in\{1, \ldots, m-1\}$, we have $\chi(n, p, q)=-1$ if $2 p \leq 2(q-m)+1$ and otherwise $\chi(n, p, q)=+1$. Finally, if $q \in\{1, \ldots, m\}$ and $(p-m) \in\{1, \ldots, m-1\}$, we have $\chi(n, p, q)=+1$ if $2 q \leq 2(p-m)+1$ and otherwise $\chi(n, p, q)=-1$.

For a general hyperline $p$ in which $(\operatorname{sign}(q), q)$ comes before $(\operatorname{sign}(r), r)$, we have $\chi(p, q, r)=\operatorname{sign}(q) \operatorname{sign}(r) . \chi$ has to be alternating on $\{1, \ldots, n\}^{3}$ and it has to be compatible with the definition in other lines, i.e., with the definition in lines $q$ and $r$. In addition we know a partial ordering on the set of signed cocircuits. For $p \in$ $\{1, \ldots, 2 m-1\}$ we have $\chi(p,(m+j) \bmod (2 m-1), q)=-\operatorname{sign}(q)$ within line $p$, and $\chi(p,(m-1+j) \bmod (2 m-1), q)=\operatorname{sign}(q)$ within line $p$. This implies in lines $(m+j) \bmod (2 m-1)$ and $(m-1+j) \bmod (2 m-1)$ an ordering of $p$ with respect to $q$, etc.

There exists an implemention of this algorithm written by the first author and S. Jost in the functional language GOFER. Our results have shown that the impression coming from small examples with up to 16 elements (where there is only one arrangement for each $n$ ) has to be replaced with what is known from Mendelsohn triple systems. Although our geometric conditions form a filter and we have many less arrangements than Mendelsohn triple systems, there are now huge classes of examples of $p_{3}$-maximal pseudoline arrangements apart from those known before via constructions like the two new ones described above. 


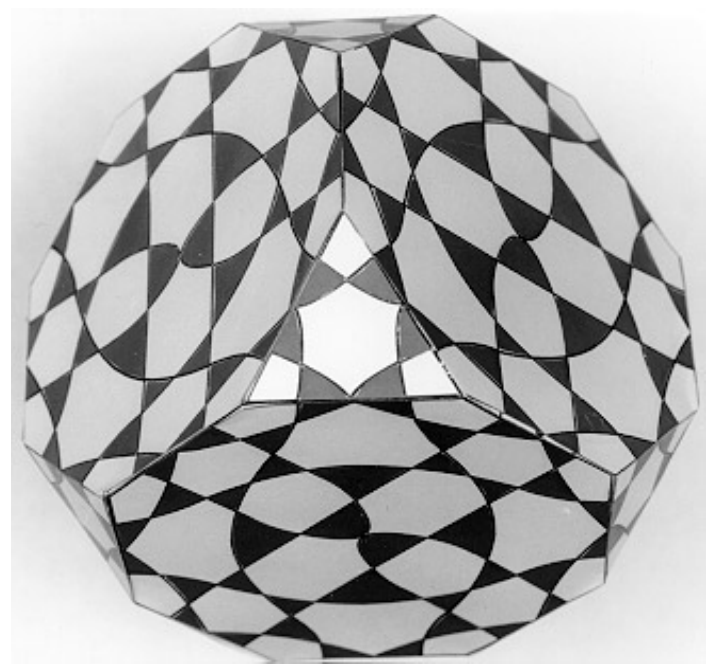

Fig. 4. The new $p_{3}$-maximal pseudoline arrangements with $n=18$ elements depicted as a one-sphere system on a truncated cube.

The program reconfirmed the uniqueness up to $n=16$ and found a first and last unknown example for $n=18$ (Fig. 4).

In the case $n=18$ we even have different $p$-vectors (Fig. 5). The $p$-vector has as its components the number of 3-gons, 4-gons, etc., of the arrangement.

For $n=22$ there exist precisely 10 examples with a symmetry of order 3 depicted in Fig. 6.
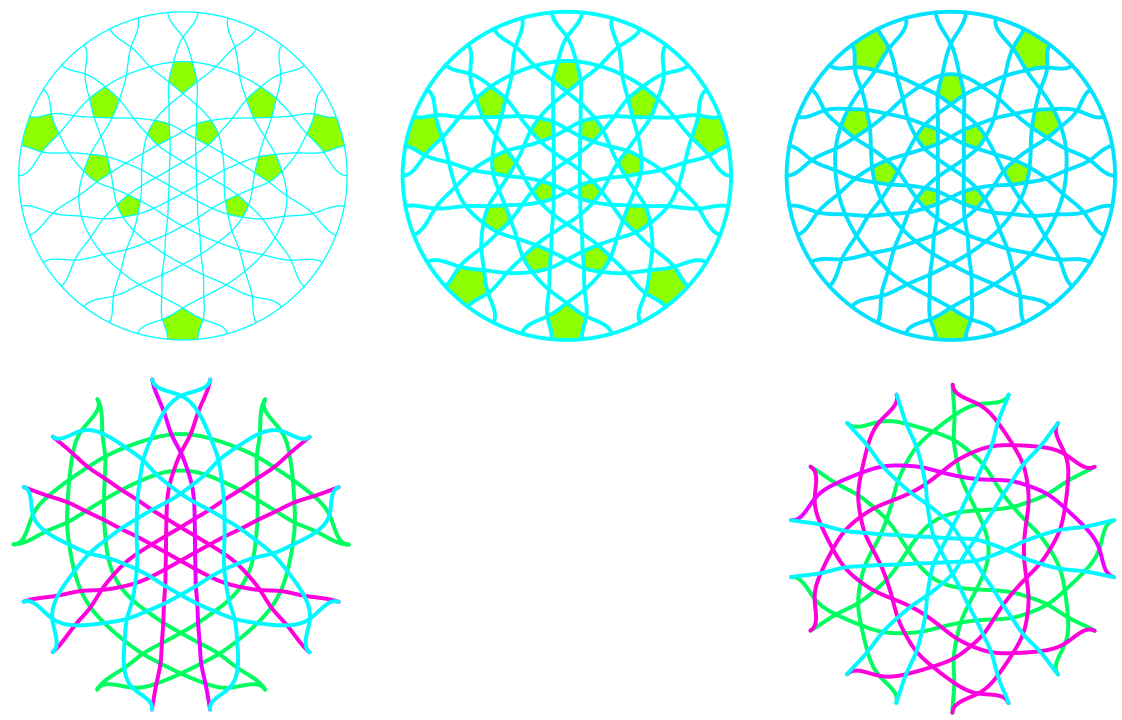

Fig. 5. $p_{3}$-maximal pseudoline arrangements with $n=18$ elements. $p$-vector left and right: $(102,0,12,34$, $6)$ and $p$-vector in the middle: $(102,0,18,22,12)$. 
$(154,0,24,36,18)$
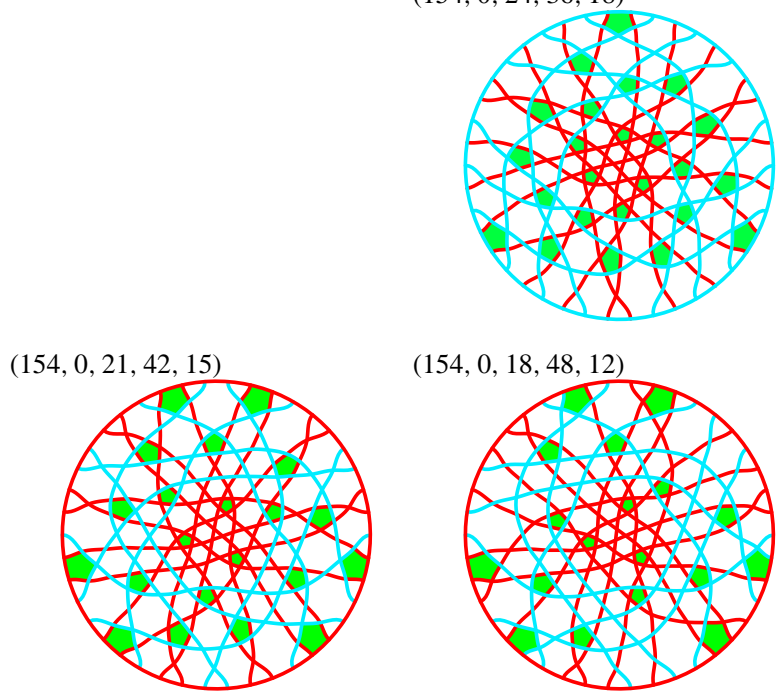

$(154,0,18,48,12)$

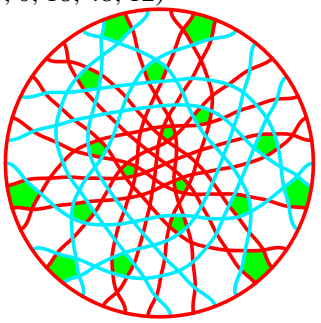

$(154,0,15,54,9)$

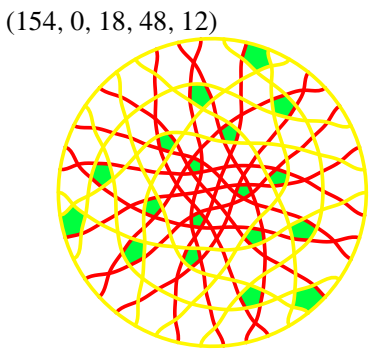

$(154,0,15,54,9)$

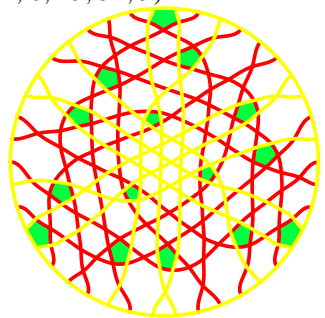

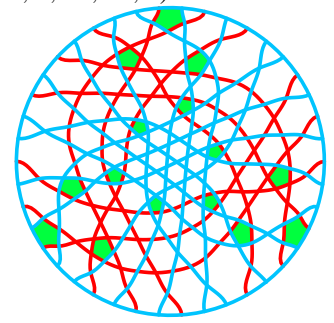

$(154,0,15,54,9)$

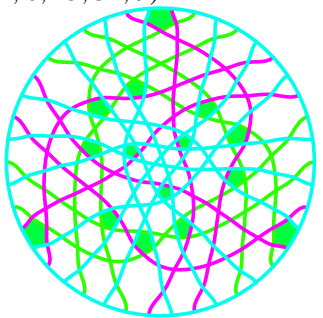

$(154,0,18,48,12)$

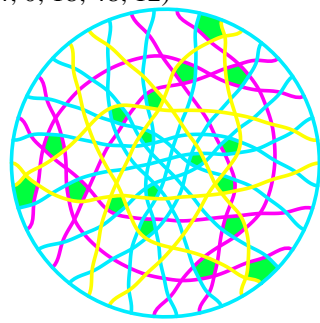

$(154,0,15,54,9)$

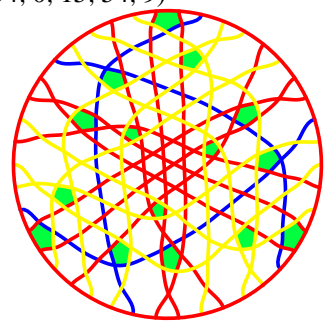

$(154,0,15,54,9)$

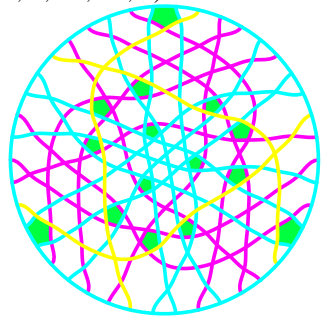

Fig. 6. All $p_{3}$-maximal pseudoline arrangements with $n=22$ elements and with a symmetry of order 3 .

The classification of all $p_{3}$-maximal pseudoline arrangements with $n=28$ elements and with a symmetry of order 3 lead to precisely 86 different examples with altogether 13 different $p$-vectors.

These new examples with 22 and 28 elements and a symmetry of order 3 helped the second author to find new recursive constructions for $n \rightarrow 7 n-6$ (three different constructions are known) and $n \rightarrow 9 n-8$ (ten different constructions are known). For details please contact the authors. 

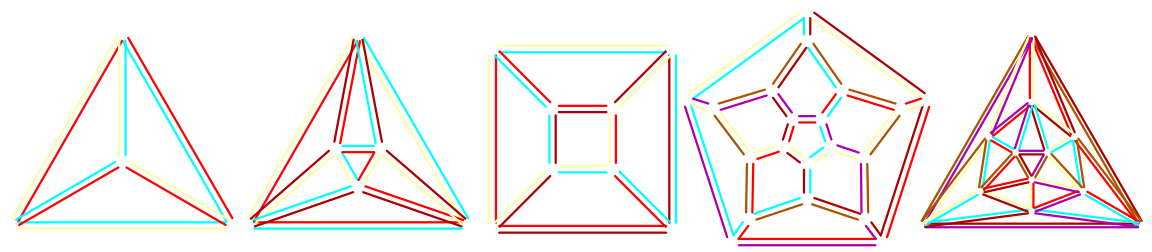

Fig. 7. Schlegeldiagrams of Platonic solids with its Petrie polygons.
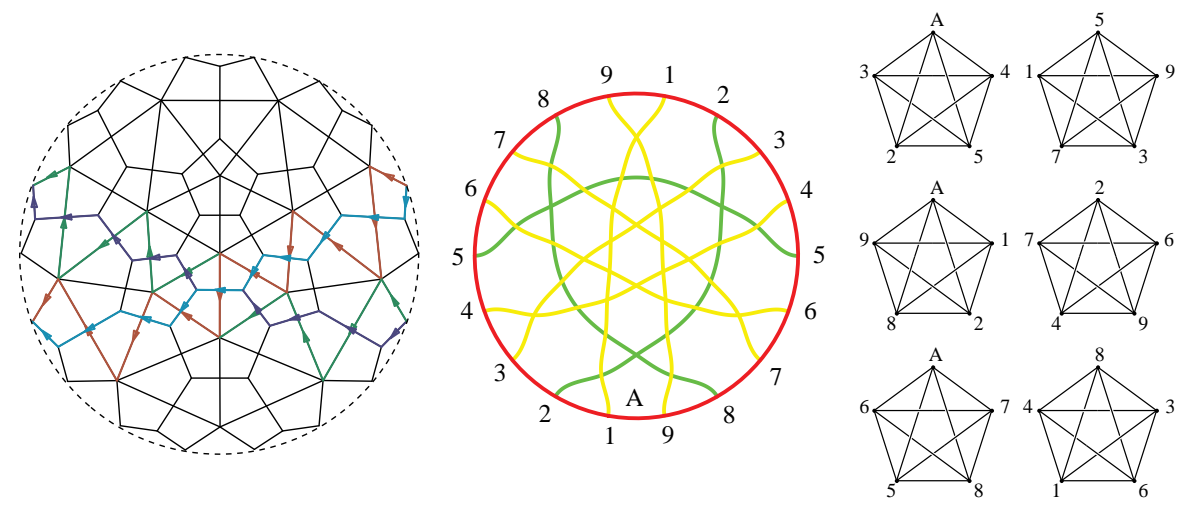

Fig. 8. Three isomorphic mathematical objects.
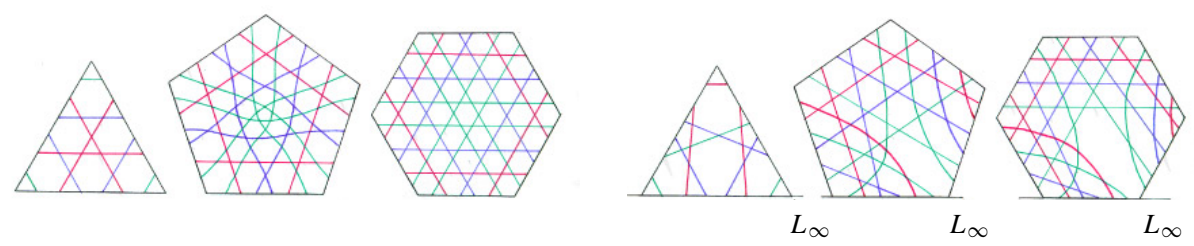

Fig. 9. Decomposition of old cells in the case $n \rightarrow 7 n-6$.
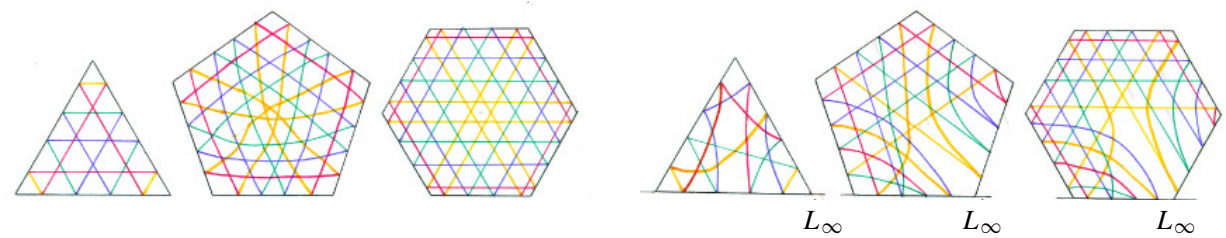

Fig. 10. Decomposition of old cells in the case $n \rightarrow 8 n-8$. 

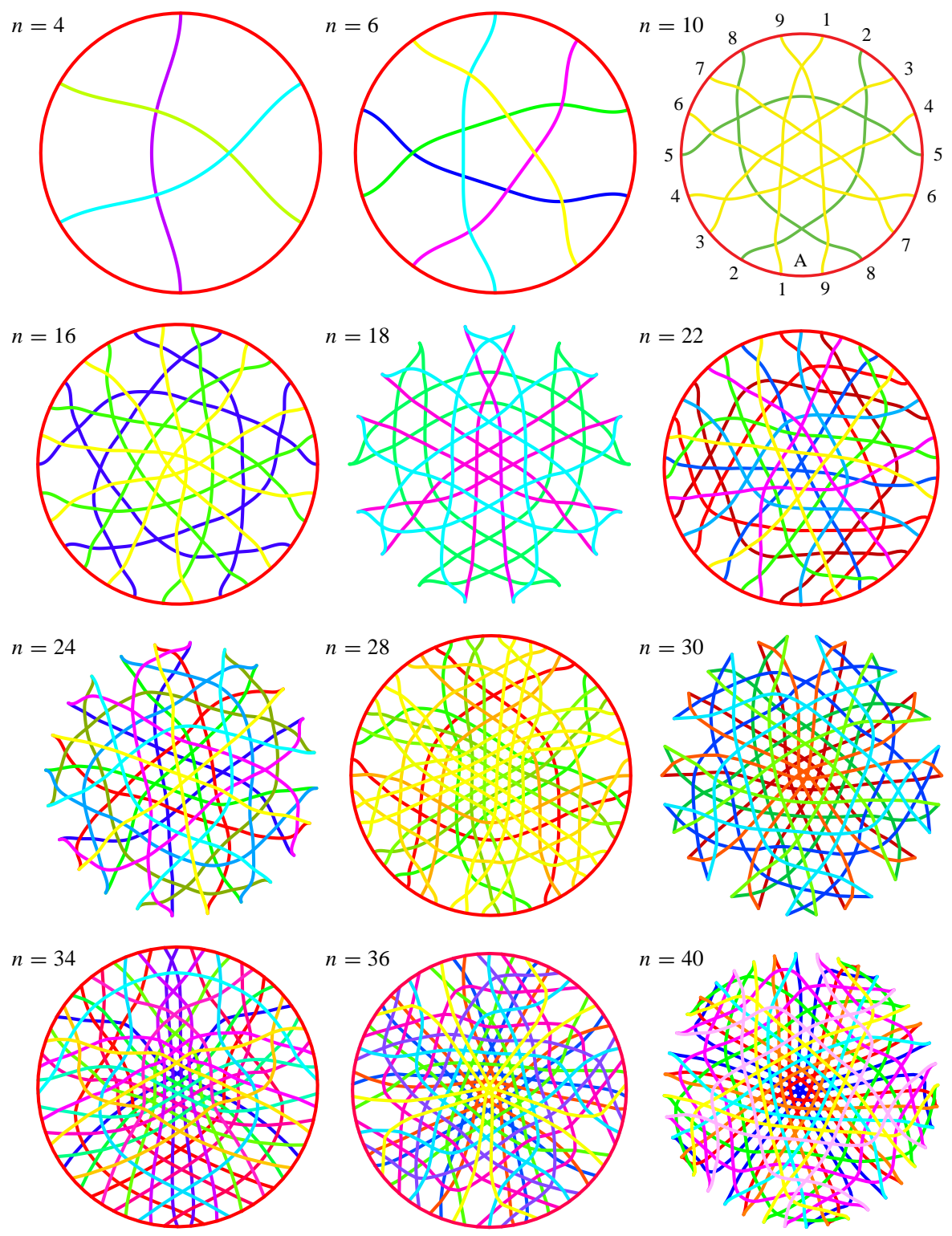

Fig. 11. $p_{3}$-maximal pseudoline arrangements with up to $n=40$ elements. 


\section{A Complete List of Existence up to $n \leq \mathbf{4 0}$}

We have provided colored pictures of at least one example for each $n \leq 40$ for which we know that there exists a $p_{3}$-maximal pseudoline arrangement. The list is complete up to $n \leq 40$. For $n=48$ we have the smallest open case. The given solutions for $n=4,6$, $10,16,18,22,24,28,30,34,36,40$ lead via various constructions like those provided in Section 3 to infinite series of $p_{3}$-maximal pseudoline arrangements.

Figure 11 describes how recursive constructions can be applied, starting with the first values of $n$. In particular, we can see how the transformations $n \rightarrow 7 n-6$ and $n \rightarrow 8 n-8$ look like globally on these examples with $22,24,36$, and 40 pseudolines.

In Fig. 12 we have depicted some additional examples, which were not obtained by constructions but via our algorithm. We see $p_{3}$-maximal pseudoline arrangements with
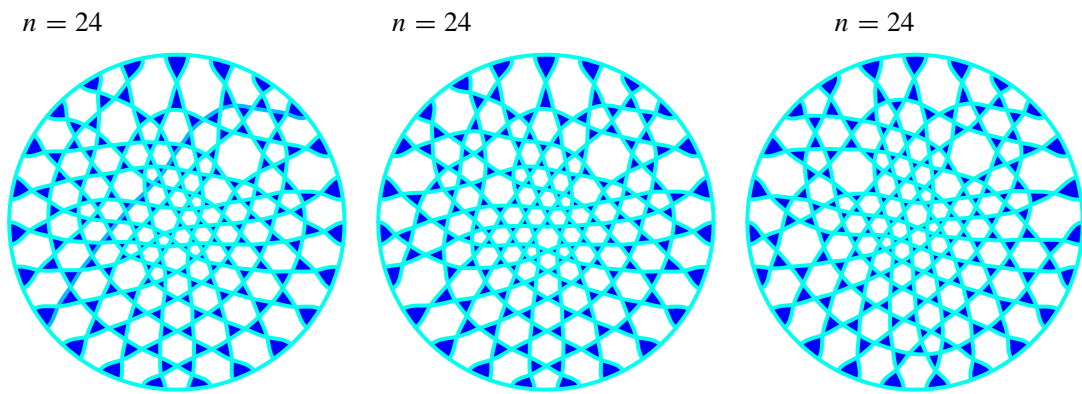

$n=28(252,0,33,70,21,3) \quad n=28(252,0,24,88,12,3)$

$n=28(252,0,21,91,15)$
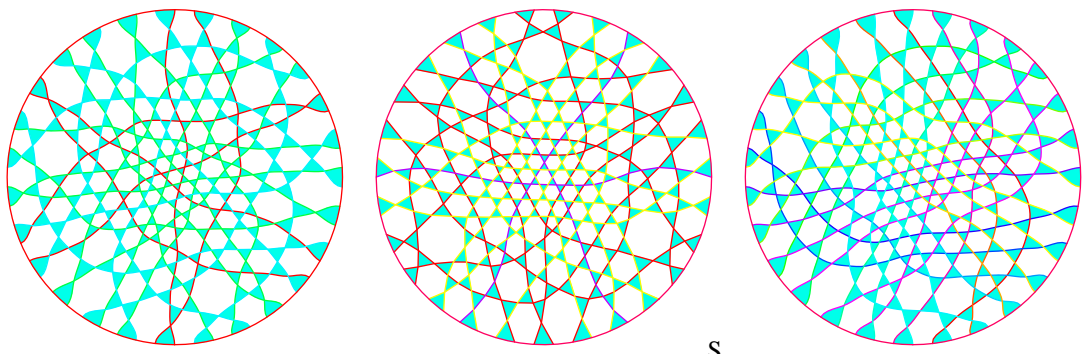

$n=36$

$n=36$
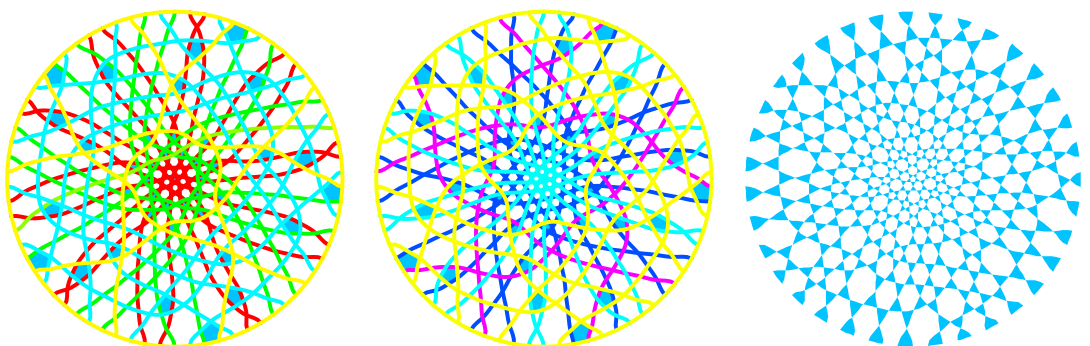

Fig. 12. Some $p_{3}$-maximal pseudoline arrangements with $n=24,28,36$ elements. 
$n=24,28,36$ elements. The three examples with 28 elements have a different $p$-vector from Harborth's example.

Remark. Several pictures of pseudoline arrangements shown in this article have been generated by the program OMAWIN. The implementation of this program was supported in part by the Deutsche Forschungsgemeinschaft (DFG). Its early version was developed with J. Richter-Gebert in connection with several open problems in the theory of oriented matroids. In the meantime it was extended very much to its present version by P. Schuchert, M. Gebhard, and D. Klodt. Additional implementations for working with oriented matroids written by A. Guedes de Oliveira, T.-K. Strempel, K. P. Pock, J. Scharnbacher, U. Thiemann and C. Engel and especially RADONCHI and CHIMAN written by P. Schuchert are available. They are useful for handling oriented matroids with special properties.

\section{References}

1. Altshuler, A., Bokowski, J., and Schuchert, P. (1996), Neighborly 2-manifolds with 12 vertices, J. Combin. Theory Ser. A, 75(1), 148-162.

2. Altshuler, A., Bokowski, J., and Schuchert, P. (1995), Spatial polyhedra without diagonals, Israel J. Math., 86, 373-396.

3. Barthel, G., Hirzebruch, F., and Höfer, T. (1987), Geradenkonfigurationen und Algebraische Flächen, Vieweg Verlag, Braunschweig.

4. Björner, A., Las Vergnas, M., Sturmfels, B., White, N., and Ziegler, G.M. (1993), Oriented Matroids, Cambridge University Press, Cambridge.

5. Bokowski, J. (1993), Oriented matroids, in Handbook of Convex Geometry (Gruber, P., and Wills, J.M., eds.), Elsvier, North-Holland, Amsterdam, pp. 555-602.

6. Bokowski, J., and Strempel, T.-K. (1995), Pseudogeraden Arrangements mit maximaler Dreiecksanzahl, Manuscript.

7. Brehm, U., and Wills, J.M. (1993), Polyhedral manifolds, in Handbook of Convex Geometry (Gruber, P., and Wills, J.M., eds.), Elsvier, North-Holland, Amsterdam, pp. 535-554.

8. Coxeter, H.S.M. (1973), Regular Polytopes, 3rd ed., Dover, New York.

9. Coxeter, H.S.M., and Moser, W.O.J. (1980), Generators and Relations for Discrete Groups, 4th edn., Springer-Verlag, Berlin.

10. Folkman, J., and Lawrence, J. (1978), Oriented matroids, J. Combin. Theory Ser. B, 25, 199-236.

11. Forge, D., and Ramirez Alfonsin, J.L. (1996), Straight line arrangements in the real projective plane, Preprint.

12. Grek A.S. (1960), Regular polyhedra on a closed surface with $\chi=-1$, Trudy Tbiliss. Mat. Inst., 27, 103-112.

13. Grünbaum B. (1972), Arrangements and Spreads, Regional Conference Proceedings, Vol. 10, American Mathematical Society, Providence, RI.

14. Harborth, H. (1985), Some simple arrangements of pseudolines with a maximum number of triangles, Discrete Geom. Conv. N.Y. Acad. Sci., 440, 30-31.

15. Las Vergnas, M. (1975), Matrö̈des orientables, C.R. Acad. Sci. Paris Ser. A, 280, 61-64.

16. Levi, F. (1926), Die Teilung der projektiven Ebene durch Gerade und Pseudogerade, Ber. Math.-Phys. Kl. Sächs. Akad. Wiss., 78, 256-267.

17. McMullen, P. (1994), Modern developments in regular polytopes, in Polytopes: Abstract, Convex and Computational (Bisztriczky, T., McMullen, P., Schneider, R., and Ivić Weiss, A., eds.), Series C, Mathematical and Physical Sciences Vol. 440, Kluwer, Dordrecht, pp. 97-124.

18. McMullen, P., and Schulte, E. (1997), Regular polytopes in ordinary space, Discrete Comput. Geom., this issue, pp. 449-478. 
19. Roudneff, J.-P. (1986), On the number of triangles in simple arrangements of pseudolines in the real projective plane, Discrete Math., 60, 234-251.

20. Roudneff, J.-P. (1987), Quadrilaterals and pentagons in arrangements of lines, Geom. Dedicata, 23, 221227.

21. Roudneff, J.-P. (1988), Arrangements of lines with a minimal number of triangles are simple, Discrete Comput. Geom., 3, 97-102.

22. Roudneff, J.-P. (1996), The maximum number of triangles in arrangements of pseudolines, J. Combin. Theory Ser. B, 66, 44-74.

23. Strempel, T.-K. (1996), Zur Erzeugung, Klassifizierung und Einbettung kombinatorischer Mannigfaltigkeiten, Ph.D. Thesis, University Darmstadt.

24. Ziegler, G. (1995), Lectures on Polytopes, Springer-Verlag, Berlin.

Received July 18, 1996, and in revised form October 28, 1996. 